\title{
Layered Orthogonal Frequency Division Multiplexing with Index Modulation
}

\author{
Jun Li, Member, IEEE, Shuping Dang, Member, IEEE, Miaowen Wen, Senior Member, IEEE, \\ Xue-Qin Jiang, Member, IEEE, Yuyang Peng, Member, IEEE, and Han Hai
}

\begin{abstract}
In this paper, we propose a novel scheme termed layered orthogonal frequency division multiplexing with index modulation (L-OFDM-IM) to increase the spectral efficiency (SE) of OFDM-IM systems. In L-OFDM-IM, all subcarriers are first divided into multiple layers, each determining the active subcarriers and their modulated symbols. The IM bits are carried on the indices of the active subcarriers of all layers, which are overlapped and distinguishable with different signal constellations so that the number of the IM bits is larger than that in traditional OFDM-IM. A low-complexity detection is proposed to alleviate the high burden of the optimal maximumlikelihood detection at the receiver side. A closed-form upper bound on the BER, the achievable rate and diversity order are derived to characterize the performance of L-OFDM-IM. To enhance the diversity performance of L-OFDM-IM, we further propose coordinate interleaving L-OFDM-IM (CI-L-OFDM-IM), which interleaves the real and imaginary parts of the modulated symbols over two different subchannels. Computer simulations verify the theoretical analysis, whose results show that L-OFDMIM outperforms the conventional OFDM-IM scheme. Moreover, it is also confirmed that CI-L-OFDM-IM obtains an additional diversity order in comparison with L-OFDM-IM.
\end{abstract}

Index Terms-Orthogonal frequency division multiplexing (OFDM), index modulation, bit error rate (BER), diversity, maximum-likelihood (ML).

\section{INTRODUCTION}

I NDEX modulation (IM), as a promising modulation technique for the fifth generation $(5 \mathrm{G})$ communication systems, makes good use of the index(es) to transmit additional information bits [1], [2]. The index(es) media can be transmit or receive antennas, orthogonal frequency division multiplexing (OFDM) subcarriers, time slots, linear block codes and so on [3], [4]. Since IM only utilizes partial resource to transmit signals, it leads to a high energy efficiency (EE).

This work was supported in part by National Nature Science Foundation of China under Grants 61701127, 61871190, 61671143 and 61801106, in part by the Natural Science Foundation of Guangdong Province under Grant 2018B030306005, and in part by the Pearl River Nova Program of Guangzhou under Grant 201806010171. (Corresponding author: Miaowen Wen.)

$\mathrm{J}$. $\mathrm{Li}$ is with the School of Mechanical and Electrical Engineering, Guangzhou University, Guangzhou 510006, China (e-mail: lijun52018@gzhu.edu.cn).

S. Dang was with Department of Engineering Science, University of Oxford when submitting the first version of this paper, and is now with Computer, Electrical and Mathematical Sciences and Engineering Division, King Abdullah University of Science and Technology (KAUST), Thuwal 23955-6900, Saudi Arabia (e-mail: shuping.dang@kaust.edu.sa).

M. Wen is with the School of Electronic and Information Engineering, South China University of Technology, Guangzhou 510641, China (e-mail: eemwwen@scut.edu.cn).

X.-Q. Jiang and H. Hai are with the School of Information Science and Technology, Donghua University, Shanghai 201620, China (e-mail: \{xqjiang, hhai\}@dhu.edu.cn).

Y. Peng is with the Faculty of Information Technology, Macau University of Science and Technology, Taipa , Macau (e-mail: yypeng@must.edu.mo).
The first attempt of applying the IM concept to the space domain refers to the spatial modulation (SM) [5], which utilizes the index of a single active antenna to convey extra information bits. Since only one transmit antenna is active, SM obtains several advantages, for instance, a high EE, a single radio frequency (RF) chain and no inter-channel interference. To further increase the EE of SM systems, space shift keying (SSK) is proposed to convey information bits only through the active antenna [6]. Although both SM and SSK achieve a high EE, the spectral efficiency (SE) of SM/SSK systems is relatively low due to the uncirculated antennas. Therefore, many studies aim to improve the SE of SM/SSK systems [7], [8]. To increase the SE of SM systems, generalised SM (GSM) is proposed to activate multiple transmit antennas in each transmission phase [7]. In a similar manner, generalized SSK (GSSK) is proposed in [8], which can achieve a higher SE than that of SSK. Another efficient scheme, named quadrature SM (QSM), is proposed to improve the SE of SM systems by splitting the IM bits into in-phase (I-) and quadrature (Q-) domains [9], [10]. Recently, layered SM (LSM) is proposed to increase the SE of SM systems by grouping transmit antennas [11]. Similarly, layered SSK (LSSK) is proposed to divide the transmit antennas into multiple layers so as to form a layer domain, by which a higher SE is achievable [12]. In addition to the SE enhancement, a variety of derivative SM schemes has been proposed to achieve the diversity gain for SM/SSK by incorporating SM into other domains [13]-[16]. In [13] and [14], space-time block coded SM (STBC-SM) and space-time shift keying (STSK) are proposed to enhance the diversity order of the conventional SM/SSK scheme. Differential SM (DSM) is an extension of SM into space and time domains with no need of channel state information (CSI) at both transmitting and receiving sides [15]. Benefiting from the advantages of DSM, space-time block coded DSM (STBCDSM) is then proposed to acquire the diversity enhancement without CSI [16].

Besides, applying the IM concept into the frequency domain is first investigated in [17] and [18]. In [17], subcarrier-index modulation OFDM (SIM-OFDM) is proposed to utilize the index(es) of subcarriers to transmit the IM bits in OFDM systems. Enhanced SIM-OFDM (ESIM-OFDM) is then proposed to achieve a better performance than SIM-OFDM [18]. Nevertheless, the SE of SIM-OFDM and ESIM-OFDM is very low compared to the conventional OFDM scheme. To solve this problem, OFDM with IM (OFDM-IM) is proposed to flexibly transmit IM bits by the indices of active subcarriers [19], [20]. Specifically, only a subset of the whole subcarriers 
are activated to carry conventional modulation (CM) symbols and the indices of the active subcarriers are utilized to map the additional information bits (i.e. IM bits). Different mapping rules between IM bits and indices of the active subcarriers, e.g. the combinatorial rule [19] and the equiprobable subcarrier activation rule [21] as well as the on-off keying (OOK) activation rule [22], [23], have different impacts on the BER performance of OFDM-IM. Although OFDM-IM achieves a better BER performance, the SE of OFDM-IM is usually lower [24]. Therefore, many research studies aim to improve the SE of OFDM-IM systems. For example, OFDM with I/Q IM (OFDM-IM-IQ) is proposed to enhance the SE of OFDM-IM systems by performing the IM concept into IQ domains [25]. OFDM with generalized index modulation (OFDM-GIM) is also proposed to engthen the IM bit sequence [26]. Besides, OFDM-IM is combined with multiple-input multiple-output (MIMO) systems to form MIMO-OFDMIM, which considerably raises the SE of OFDM-IM systems [27]. In [28], dual-mode IM aided OFDM (DM-OFDM) is tactfully designed to transmit the modulated symbols through not only active subcarriers but also "inactive" subcarriers, which successfully enhances the SE of OFDM-IM systems. To be general, multiple-mode OFDM-IM (MM-OFDM-IM) is developed to transmit modulated symbols through all subcarriers and the IM bits are carried by the permutations of multiple distinguishable constellations [29]-[31]. A diversity enhancement mechanism of MM-OFDM-IM is investigated in [32]. In addition, the OFDM-IM technique is widely applied into relay and vehicular communications [33], [34].

In this paper, we propose a novel OFDM-IM scheme, named layered-OFDM-IM (L-OFDM-IM) to increase the SE of OFDM-IM systems without any additional expense. Unlike OFDM-IM, L-OFDM-IM splits the indices of all subcarriers into $L$ layers and each layer only contains partial and overlapping subcarriers (a.k.a. candidate subcarriers). More explicitly, the active subcarriers are selected from their own candidate sets at each layer, in which the selected subcarriers carry the modulated symbols generated from carefully designed distinguishable constellations. Accordingly, the IM bits of LOFDM-IM are represented by all active subcarriers in $L$ layers. By properly choosing the candidate subcarriers in each layer, the proposed L-OFDM-IM scheme is capable of increasing the length of IM bits, and results in an improved SE compared to OFDM-IM. A low-complexity detection method is proposed to relieve the high computational complexity of the optimal maximum likelihood (ML) detection. We then study the BER performance of L-OFDM-IM and derive an upper bound on the BER according to the union bounding technique. The achievable rate and diversity order of L-OFDM-IM are also characterized. To provide a diversity gain, we further propose the coordinate interleaving L-OFDM-IM (CI-L-OFDM-IM) scheme, which interleaves the real and imaginary parts of the modulated symbols over two different subcarriers (or subchannels). Simulation results show that the proposed LOFDM-IM outperforms the conventional OFDM-IM scheme with the same diversity order. In addition, CI-L-OFDM-IM is verified to harvest an additional diversity order.

The rest of this paper is organized as follows. In Section II,

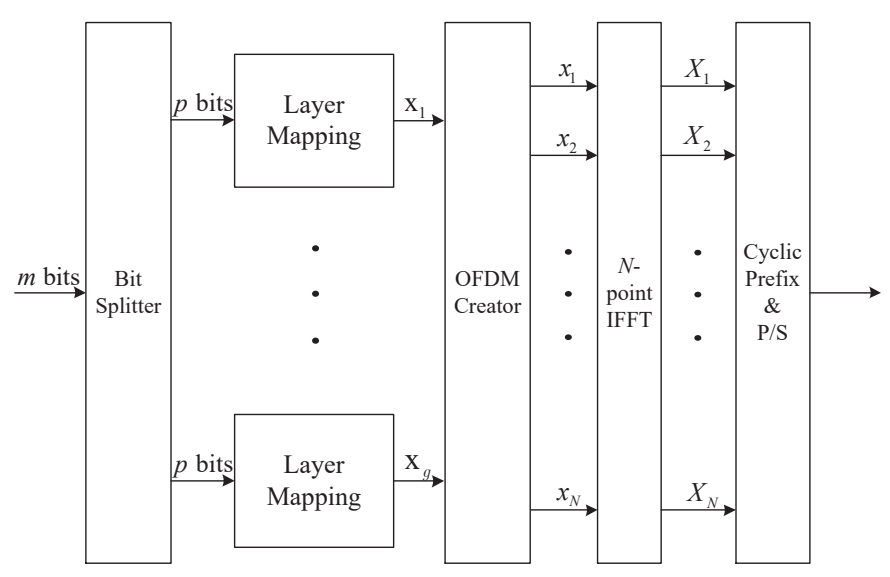

Fig. 1. System model for L-OFDM-IM.

we introduce the system model of L-OFDM-IM. Section III derives the BER upper bound and achievable diversity order of L-OFDM-IM systems. Simulation results are presented and discussed in Section IV. Finally, this paper is concluded in Section V. ${ }^{1}$

\section{PROPOSED L-OFDM-IM}

\section{A. System Model}

In this subsection, we propose a novel OFDM-IM scheme, namely L-OFDM-IM, which extends the length of IM bits so as to enhance the SE of OFDM-IM systems.

Fig. 1 shows the system model of L-OFDM-IM. In LOFDM-IM, the OFDM block corresponding to $N$ subcarriers is divided into $g$ groups, each consisting of $n$ subcarriers and obviously we have $n=N / g$. The incoming equiprobable bit sequence with length $m$ are also split into $g$ groups and $p$ bits are allocated to each group with $m=p g$. Since all groups are statistically independent and identical as a fundamental property of OFDM with sufficiently long cyclic prefix (CP), in the following we only take into account the $\alpha$-th group $(\alpha \in\{1,2, \ldots, g\}$ ) with $p$ bits and $n$ subcarriers for analysis, which is depicted in Fig. 2. The $p$ bits are first divided into $L$ segments pertaining to $L$ layers (this procedure is termed as layer mapping) with $p=p_{1}+p_{2}+\ldots+p_{L}$. Assume that $t$ subcarriers are utilized in each layer. At the $u$-th layer with $u \in\{1,2, \ldots, L\}, p_{u}$ information bits are further divided into two segments with $p_{u 1}$ and $p_{u 2}$ bits, where the former is defined as IM bits and the latter as CM bits. The IM bits $\left(p_{u 1}=\left\lfloor\log _{2} C(t, k)\right\rfloor\right)$ are used to select $k$ out of $t$ subcarriers, while the modulation bits $\left(p_{u 2}=k \log _{2} M\right)$ are used to

\footnotetext{
${ }^{1}$ Upper and lower case boldface letters denote matrices and column vectors, respectively. The complex number field is represented by $\mathbb{C} .(\cdot)^{T}$ and $(\cdot)^{H}$ represent the transpose and Hermitian transpose operations, respectively. $\mathbf{I}_{M}$ is an $M \times M$ identity matrix. $\mathfrak{R}\{\cdot\}$ and $\mathfrak{J}\{\cdot\}$ represent the real and imaginary components of the argument, respectively. $E\{\cdot\}$ denotes the expectation operation. $\|\cdot\|$ denotes the Frobenius norm operation of the argument. $C(\cdot, \cdot)$ represent the binomial operation of the argument. $Q(\cdot)$ denotes the Gaussian $Q$-function. $\operatorname{rank}\{\cdot\}$ indicates the rank of the argument. $\operatorname{diag}\{\mathbf{x}\}$ denotes a diagonal matrix whose diagonal elements are drawn from $\mathbf{x} \cdot \operatorname{det}\{\cdot\}$ represents the determinant of the argument. The probability of an event is denoted by $\operatorname{Pr}(\cdot) .\lfloor\cdot\rfloor$ indicates the floor operation. $\boldsymbol{a} \backslash \boldsymbol{b}$ denotes the complementary operation that excludes set $\boldsymbol{b}$ from set $\boldsymbol{a}$.
} 


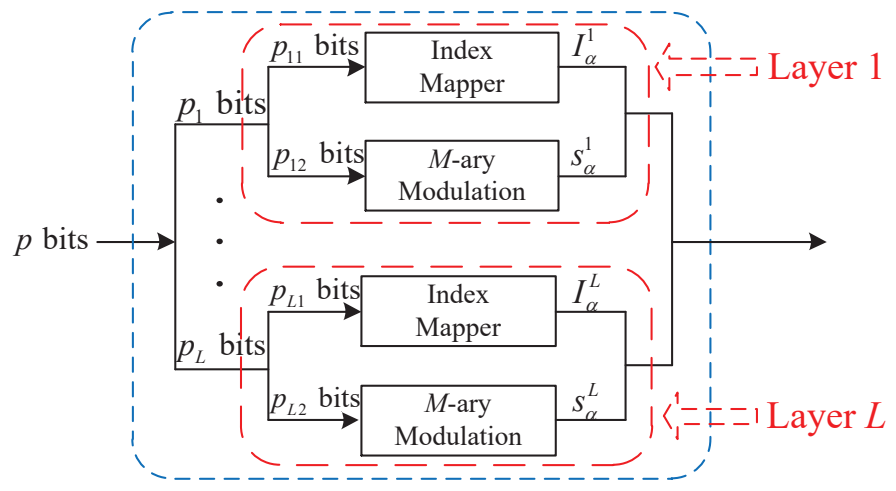

Fig. 2. Specific configuration of layer mapping for the $\alpha$-th group.

generate $k$ modulated symbols $\mathbf{s}_{\alpha}^{u}=\left[s_{\alpha}^{u, 1}, s_{\alpha}^{u, 2}, \ldots, s_{\alpha}^{u, k}\right]$, $s_{\alpha}^{u, \tau} \in \mathcal{S}_{\alpha}^{u}$ with $\tau=1,2, \ldots, k$, where $\mathcal{S}_{\alpha}^{u}$ represents the $M$ ary constellation. It is worth noting that in order to identify modulated symbols in all $L$ layers, all signal constellation sets $\left\{\mathcal{S}_{\alpha}^{u}\right\}_{u=1}^{L}$ must be distinguishable from each other. As a consequence, it is obvious that we have $n=t+k(L-1)$ and $p=L\left(\left\lfloor\log _{2} C(t, k)\right\rfloor+k \log _{2} M\right)$.

\section{B. Layer Mapping}

Remember that $p$ bits are split into $L$ segments for layer mapping purposes in each group (see Fig. 2). We take the $\alpha$ th group as an example and the details of layer mapping are explained as follows:

1) Step 1 (Layer 1 with $p_{1}$ bits): The initial subcarrier set is given by $\Theta_{\alpha}=\{1,2, \ldots, n\}$. We first take a subset of $\Theta$ as a candidate set $\Theta_{\alpha}^{1}=\{1,2, \ldots, t\}$ for layer 1 . The active subcarrier pattern (SAP) is selected from $\Theta_{\alpha}^{1}$ by $p_{11}$ bits as $I_{\alpha}^{1}=\left\{i_{\alpha}^{1,1}, i_{\alpha}^{1,2}, \ldots, i_{\alpha}^{1, k}\right\}$, where $i_{\alpha}^{1, \tau} \in \Theta_{\alpha}^{1}$ with $\tau=$ $1,2, \ldots, k$. The $k$ modulated symbols generated by $p_{12}$ bits are given by $s_{\alpha}^{1}=\left[s_{\alpha}^{1,1}, s_{\alpha}^{1,2}, \ldots, s_{\alpha}^{1, k}\right]$, where $s_{\alpha}^{1, \tau} \in \mathcal{S}_{\alpha}^{1}$. Then, the resulting signal vector for layer 1 can be expressed as

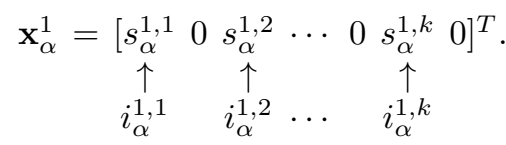

2) Step 2 (Layer 2 with $p_{2}$ bits): The candidate set for layer 2 is updated as $\Theta_{\alpha}^{2}=\{1,2, \ldots, t, t+1, \ldots, t+$ $k\} \backslash I_{\alpha}^{1}$. The SAP is selected from $\Theta_{\alpha}^{2}$ by $p_{21}$ bits as $I_{\alpha}^{2}=$ $\left\{i_{\alpha}^{2,1}, i_{\alpha}^{2,2}, \ldots, i_{\alpha}^{2, k}\right\}$, where $i_{\alpha}^{2, \tau} \in \Theta_{\alpha}^{2}$. The $k$ modulated symbols obtained from $p_{22}$ bits for layer 2 are expressed by $s_{\alpha}^{2}=\left[s_{\alpha}^{2,1}, s_{\alpha}^{2,2}, \ldots, s_{\alpha}^{2, k}\right]$, where $s_{\alpha}^{2, \tau} \in \mathcal{S}_{\alpha}^{2}$. The resulting $n \times 1$ vector is then given by

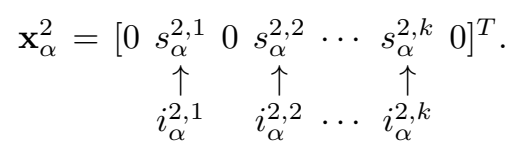

3)Step 3: The above two steps are repeated until all resulting vectors of $L$ layers are obtained as $\left\{\mathbf{x}_{\alpha}^{1}, \mathbf{x}_{\alpha}^{2}, \ldots, \mathbf{x}_{\alpha}^{L}\right\}$. Therefore, the signal vector for the $\alpha$-th group is given by

$$
\begin{aligned}
\mathbf{x}_{\alpha} & =\left[\mathbf{x}_{\alpha}^{1}+\mathbf{x}_{\alpha}^{2}+\cdots+\mathbf{x}_{\alpha}^{L}\right] \\
& =\left[x_{(\alpha-1) n+1}, x_{(\alpha-1) n+2}, \ldots, x_{(\alpha-1) n+n}\right]^{T} .
\end{aligned}
$$

For clarity and illustration purposes, we give an example of layer mapping ( $\alpha$-th layer) with $n=8, t=6, k=2, L=2$ and $M=2$. Assume the information bits $\left[\begin{array}{lllllllll}1 & 1 & 0 & 1 & 1 & 0 & 0 & 1 & 1\end{array}\right]$ with $p=10$ are transmitted over one time instant and two distinguishable constellation sets for layer 1 and layer 2 are given as $\mathcal{S}_{\alpha}^{1}=\{-11\}$ and $\mathcal{S}_{\alpha}^{2}=\{-j j\}$, respectively. The first 5 bits $\left(p_{1}=5\right)\left[\begin{array}{lllll}1 & 1 & 0 & 1 & 1\end{array}\right]$ are used for layer 1 and the candidate subcarrier set is $\Theta_{\alpha}^{1}=\{1,2,3,4,5,6\}$. Specifically, $\left\lfloor\log _{2} C(6,2)\right\rfloor$ bits $\left(p_{11}=3\right)\left[\begin{array}{lll}1 & 1 & 0\end{array}\right]$ indicate the SAP $I_{\alpha}^{1}=\{2,4\}$ and $2 \log _{2} M$ bits $\left(p_{12}=2\right)[11]$ are mapped into $s_{\alpha}^{1}=[1,1]$. Then, the resulting vector is obtained by $\mathbf{x}_{\alpha}^{1}=[0,1,0,1,0,0,0,0]^{T}$. The second 5 bits $\left(p_{2}=5\right)$ $\left[\begin{array}{lllll}0 & 0 & 1 & 1 & 1\end{array}\right]$ are used for layer 2 and the candidate subcarrier set is updated as $\Theta_{\alpha}^{2}=\{1,2,3,4,5,6,7,8\} \backslash\{2,4\}=$ $\{1,3,5,6,7,8\}$. The subsequent 3 bits $\left(p_{21}=3\right)\left[\begin{array}{lll}0 & 0 & 1\end{array}\right]$ indicate the $\operatorname{SAP} I_{\alpha}^{2}=\{1,5\}$ and 2 bits $\left(p_{22}=2\right)$ $\left[\begin{array}{ll}1 & 1\end{array}\right]$ are mapped into $\boldsymbol{s}_{\alpha}^{1}=[j, j]$. Then, the resulting vector is obtained by $\mathbf{x}_{\alpha}^{2}=[j, 0,0,0, j, 0,0,0]^{T}$. Finally, the transmitting signal vector for the $\alpha$-th group is given by $\mathbf{x}_{\alpha}=\mathbf{x}_{\alpha}^{1}+\mathbf{x}_{\alpha}^{2}=[j, 1,0,1, j, 0,0,0]^{T}$. It is worth noting that the second constellation set $\mathcal{S}_{\alpha}^{2}$ is generated by shifting $\pi / 2$ of the first constellation set $\mathcal{S}_{\alpha}^{1}$ in the above example. More generally for a large value of $L$, each constellation set is generated by shifting $\frac{2 \pi}{L M}$ from the previous constellation set, where the first constellation set is assumed to be the conventional phase shift keying (PSK) set.

\section{L-OFDM-IM Transceiver}

After obtaining the transmitting signal vectors for all groups, the OFDM signal vector in the frequency-domain can be expressed as

$$
\mathbf{x}_{\text {sum }}=\left[\mathbf{x}_{1}^{T}, \mathbf{x}_{2}^{T}, \ldots, \mathbf{x}_{g}^{T}\right]^{T}=\left[x_{1}, x_{2}, \ldots, x_{N}\right]^{T} .
$$

The remaining process is the same as the conventional OFDM scheme. An $N$-point IFFT is applied into the OFDM signal vector $\mathbf{x}_{\text {sum }}$ and the resulting time-domain signal vector is obtained by $\mathbf{X}_{\text {sum }}=\left[X_{1}, X_{2}, \ldots, X_{N}\right]^{T}$. Appending a sufficiently long $\mathrm{CP}$ and parallel/serial $(\mathrm{P} / \mathrm{S})$ to $\mathbf{X}_{\text {sum }}$, the OFDM signal vector is ready to be transmitted.

At the receiver side, the corresponding frequency-domain signal vector can be expressed as

$$
\mathbf{y}=\operatorname{diag}\{\mathbf{h}\} \mathbf{x}_{\text {sum }}+\mathbf{w},
$$

where $\mathbf{h}=\left[h_{1}, h_{2}, \ldots, h_{N}\right]^{T} ; h_{\iota}$ with $\iota \in\{1,2, \ldots, N\}$ is the channel coefficient following the complex Gaussian distribution with mean 0 and unit variance, and the entries of $\mathbf{w}$ follow the complex Gaussian distribution with mean 0 and variance $N_{0}$.

The optimal ML detection can be implemented in a groupwise manner owing to the independence among all subblocks (groups) and the detection criterion is given infra:

$$
\left(\left\{\hat{I}_{\alpha}^{\varphi}\right\}_{\varphi=1}^{L},\left\{\hat{\mathbf{s}}_{\alpha}^{\varphi}\right\}_{\varphi=1}^{L}\right) \underset{\left\{I_{\alpha}^{\varphi}\right\}_{\varphi=1}^{L},\left\{s_{\alpha}^{\varphi}\right\}_{\varphi=1}^{L}}{\arg \min }\left\|\mathbf{y}_{\alpha}-\operatorname{diag}\left\{\mathbf{h}_{\alpha}\right\} \mathbf{x}_{\alpha}\right\|^{2} .
$$

It should be noted that to implemente the detection criterion given in (6), the received signal and the channel coefficient vectors are correspondingly divided into $g$ groups as $\mathbf{y}=$ 
TABLE I

COMPARISONS OF IM BITS BETWEEN L-OFDM-IM AND OFDM-IM WITH DIFFERENT SYSTEM CONFIGURATIONS.

\begin{tabular}{|c||c||c||c|}
\hline$(k, t, L)$ & $\begin{array}{c}\text { IM bits } \\
\text { (L-OFDM-IM) }\end{array}$ & $(v, n)$ & $\begin{array}{c}\text { IM bits } \\
(\text { OFDM-IM) }\end{array}$ \\
\hline \hline$(1,3,2)$ & 2 bits & $(2,4)$ & 2bits \\
\hline$(2,4,2)$ & 4 bits & $(4,6)$ & 3bits \\
\hline$(2,6,2)$ & 6 bits & $(4,8)$ & 6bits \\
\hline$(2,6,3)$ & 9 bits & $(6,10)$ & 7 bits \\
\hline$(3,4,3)$ & 6 bits & $(9,10)$ & 3bits \\
\hline$(3,7,4)$ & 20 bits & $(12,16)$ & 10bits \\
\hline
\end{tabular}

$\left[\mathbf{y}_{1}^{T}, \mathbf{y}_{2}^{T}, \ldots, \mathbf{y}_{g}^{T}\right]^{T}$ and $\mathbf{h}=\left[\mathbf{h}_{1}^{T}, \mathbf{h}_{2}^{T}, \ldots, \mathbf{h}_{g}^{T}\right]^{T}$, respectively. After obtaining all SAPs and modulated symbols, $m$ information bits can be easily recovered by the demapping of SAPs and demodulation of the modulated symbols.

The SE of L-OFDM-IM systems is given by

$$
\begin{aligned}
S E_{\mathrm{L}-\text { OFDM-IM }} & =\frac{p_{1}+p_{2}+\cdots+p_{L}}{n} \\
& =\frac{L\left\lfloor\log _{2} C(t, k)\right\rfloor}{n}+\frac{L k}{n} \log _{2} M .
\end{aligned}
$$

Assume that $v$ out of $n$ subcarriers are activated in the conventional OFDM-IM scheme. In order to provide a fair comparison of SE between L-OFDM-IM and OFDM-IM, we list the number of IM bits of both L-OFDM-IM and OFDMIM schemes under the same configuration $(L k=v)$ in Table. I. Recall that the number of subcarriers in each group is $n=t+k(L-1)$ in the L-OFDM-IM scheme. It can be seen from Table I that the proposed L-OFDM-IM scheme achieves a higher SE than that of OFDM-IM scheme except the first and third comparisons with 2 and 6 IM bits. Compared to OFDM-IM, L-OFDM-IM obtains no SE gain by increasing $t$ or $M$ as revealed by the second and third comparisons, while L-OFDM-IM obtains a slight SE gain by increasing $k$ (refer to the fourth and fifth comparisons). More importantly, the proposed L-OFDM-IM scheme achieves a much larger SE gain than that of OFDM-IM scheme by increasing $L$, according to the third, fifth and sixth comparisons. Finally, from the observations above, it can be summarized that L-OFDM-IM obtains a higher SE compared to OFDM-IM with the same configuration in most cases and the conventional OFDM-IM is a special case of L-OFDM-IM with $L=1$.

\section{Low-complexity Detection}

The optimal ML detection results in a high detection burden due to overall search over all possible transmit vectors in (6). Therefore, we further propose a low-complexity detection, which largely reduces the computational complexity, especially for a large value of $n$.

We still take the $\alpha$-th group for analysis. Note that not all subcarriers are activated in each group and the last $(n-t-k(u-1))$ subcarriers will not be activated for the $u$-th layer with $u=1,2, \ldots, L$. For instance, if we have $k=2$, $t=4$ and $L=3(n=t+k(L-1)=8)$, the last four subacrriers $(5,6,7,8)$ are idle for the 1st layer and the last two subcarriers $(7,8)$ are still idle for the 2 nd layer. In this case, we first construct an Euclidean distance matrix for all subcarriers and layers excluding the idle subcarriers at each layer, which is shown at the top of the next page, where $d_{\varrho, u}$ and $\hat{x}_{\varrho, u}$ with $\varrho=1,2, \ldots, n$ and $u=1,2, \ldots, L$ denote the minimum Euclidean distance and the estimated signal for the $\varrho$-th subcarrier employing the modulation set $\mathcal{S}_{\alpha}^{u}$. Note that $d_{\varrho, u}$ and $\hat{x}_{\varrho, u}$ are calculated by

$$
\left[d_{\varrho, u}, \hat{x}_{\varrho, u}\right]=\underset{x \in \mathcal{S}_{\alpha}^{u}}{\arg \min }\left\|\mathbf{y}_{\alpha}(\varrho)-\mathbf{h}_{\alpha}(\varrho) x\right\|^{2} .
$$

The variable $d_{\varrho, 0}$, which is located in the last column of $\mathbf{D}$, denotes the Euclidean distance for the inactive subcarriers and is calculated by $d_{\varrho, 0}=\left\|\mathbf{y}_{\alpha}(\varrho)\right\|^{2}$. The symbol Inf represents the infinite Euclidean distance, which means the corresponding subcarrier is inactive and no modulated signal is estimated.

By ignoring the estimated signals and Euclidean distances for inactive subcarriers (the last column of $\mathbf{D}$ ), we then sort all Euclidean distances in $\mathbf{D}$ in ascending order in the column direction and a new matrix containing the positions of $\mathbf{D}$ is given by

$$
\mathbf{D}_{o}=\operatorname{sort}\{\mathbf{D}\}=\left[\begin{array}{ccccc}
a_{1,1} & a_{1,2} & a_{1,3} & \cdots & a_{1, L} \\
a_{2,1} & a_{2,2} & a_{2,3} & \cdots & a_{2, L} \\
\vdots & \vdots & \vdots & \ddots & \vdots \\
a_{t, 1} & a_{t, 2} & a_{t, 3} & \cdots & a_{t, L} \\
\text { Inf } & a_{t+1,2} & a_{t+1,3} & \cdots & a_{t+1, L} \\
\vdots & \vdots & \vdots & \ddots & \vdots \\
\text { Inf } & a_{t+k, 2} & a_{t+k, 3} & \cdots & a_{t+k, L} \\
\text { Inf } & \text { Inf } & a_{t+k+1,3} & \cdots & a_{t+k+1, L} \\
\vdots & \vdots & \vdots & \ddots & \vdots \\
\text { Inf } & \text { Inf } & \text { Inf } & \cdots & a_{n, L}
\end{array}\right],
$$

where $a_{1, u}$ and $a_{n, u}$ indicate the positions of the minimal value and the largest value in the $u$-th column, respectively. In other words, it is shown that the $a_{1, u}$ th and $a_{n, u}$ th subcarriers are the most and the least likely to be activated at the $u$-th layer, respectively. Therefore, we only take consideration of the half of rows in $\mathbf{D}_{o}$ as

$$
\tilde{\mathbf{D}}_{o}=\left[\begin{array}{ccccc}
a_{1,1} & a_{1,2} & a_{1,3} & \cdots & a_{1, L} \\
a_{2,1} & a_{2,2} & a_{2,3} & \cdots & a_{2, L} \\
\vdots & \vdots & \vdots & \ddots & \vdots \\
a_{n / 2,1} & a_{n / 2,2} & a_{n / 2,3} & \cdots & a_{n / 2, L}
\end{array}\right]
$$

Specifically, we aim to find all sets of legitimate SAPs from all columns in $\tilde{\mathbf{D}}_{o}$, which are denoted as

$$
\begin{array}{ccc}
\Omega_{\alpha}^{\epsilon}=\left\{\left\{a_{1,1}, \ldots, a_{k, 1}\right\}\right. & \cdots, & \left.\left\{a_{2, L}, \ldots, a_{k+1, L}\right\}\right\}, \\
\uparrow & & \uparrow \\
\hat{I}_{\alpha, \epsilon}^{1} & \cdots & \hat{I}_{\alpha, \epsilon}^{L}
\end{array}
$$

where $\epsilon=1,2, \ldots, \zeta$ and $\zeta$ denotes the number of all legitimate SAPs. For example, if we have $k=2, t=4$, 


$$
\mathbf{D}=\left[\begin{array}{cccccc}
{\left[d_{1,1}, \hat{x}_{1,1}\right]} & {\left[d_{1,2}, \hat{x}_{1,2}\right]} & {\left[d_{1,3}, \hat{x}_{1,3}\right]} & \cdots & {\left[d_{1, L}, \hat{x}_{1, L}\right]} & d_{1,0} \\
{\left[d_{2,1}, \hat{x}_{2,1}\right]} & {\left[d_{2,2}, \hat{x}_{2,2}\right]} & {\left[d_{2,3}, \hat{x}_{2,3}\right]} & \cdots & {\left[d_{2, L}, \hat{x}_{2, L}\right]} & d_{2,0} \\
\vdots & \vdots & \vdots & \ddots & \vdots & \vdots \\
{\left[d_{t, 1}, \hat{x}_{t, 1}\right]} & {\left[d_{t, 2}, \hat{x}_{t, 2}\right]} & {\left[d_{t, 3}, \hat{x}_{t, 3}\right]} & \cdots & {\left[d_{t, L}, \hat{x}_{t, L}\right]} & d_{t, 0} \\
\text { Inf } & {\left[d_{t+1,2}, \hat{x}_{t+1,2}\right]} & {\left[d_{t+1,3}, \hat{x}_{t+1,3}\right]} & \cdots & {\left[d_{t+1, L}, \hat{x}_{t+1, L}\right]} & d_{t+1,0} \\
\vdots & \vdots & \vdots & \ddots & : & \\
\text { Inf } & {\left[d_{t+k, 2}, \hat{x}_{t+k, 2}\right]} & {\left[d_{t+k, 3}, \hat{x}_{t+k, 3}\right]} & \cdots & {\left[d_{t+k, L}, \hat{x}_{t+k, L}\right]} & d_{t+k, 0} \\
\text { Inf } & \text { Inf } & {\left[d_{t+k+1,3}, \hat{x}_{t+k+1,3}\right]} & \cdots & {\left[d_{t+k+1, L}, \hat{x}_{t+k+1, L}\right]} & d_{t+k+1,0} \\
\vdots & \vdots & \vdots & \ddots & \vdots & \vdots \\
\text { Inf } & \text { Inf } & \text { Inf } & \cdots & {\left[d_{n, L}, \hat{x}_{n, L}\right]} & d_{n, 0}
\end{array}\right],
$$

$L=3$ and

$$
\tilde{\mathbf{D}}_{o}=\left[\begin{array}{lll}
3 & 1 & 4 \\
1 & 3 & 5 \\
2 & 6 & 3 \\
4 & 5 & 7
\end{array}\right]
$$

the legitimate SAP $\Omega_{\alpha}^{1}$ is given by $\Omega_{\alpha}^{1}=$ $\{\{3,1\},\{6,5\},\{4,7\}\}$ and the complementary SAP for inactive subcarriers is given by $\bar{\Omega}_{\alpha}^{1}=\{2,8\}$. It should be noted that the first two rows of $\tilde{\mathbf{D}}_{o}$ in (13) constitute an illegitimate SAP $\{\{3,1\},\{1,3\},\{4,5\}\}$ that the 1 st and 3rd subcarriers are activated twice, which conflicts with the concept of L-OFDM-IM. After obtaining $\zeta$ legitimate SAPs, the estimated SAP is finally decided by

$$
\left\{\hat{I}_{\alpha}^{u}\right\}_{u=1}^{L}=\min _{\epsilon \in\{1, \ldots, \zeta\}}\left(\sum_{u=1}^{L}\left\|\mathbf{D}\left(\hat{I}_{\alpha, \epsilon}^{u}, u\right)\right\|+\left\|\mathbf{D}\left(\bar{\Omega}_{\alpha}^{\epsilon}, L+1\right)\right\|\right),
$$

where $\mathbf{D}\left(\hat{I}_{\alpha, \epsilon}^{u}, u\right)$ denotes a vector in the $u$-th column and $\hat{I}_{\alpha, \epsilon^{-}}^{u}$ th rows of $\mathbf{D}$. Then, the corresponding modulated symbols are straightforwardly obtained from (9). The information bits can be directly recovered by the demapping of all SAPs and demodulation of the modulated symbols group by group.

Obviously, the computational complexity of the lowcomplexity detection method has a search complexity of order $\mathcal{O}(t L M)$ while the optimal ML detection method has a search complexity of order $\left(2^{\left\lfloor\log _{2} C(t, k)\right\rfloor} L M^{k}\right)$. Compared with the ML detection in (6), the search complexity of the lowcomplexity detection is greatly reduced. To be more specific, we measure the computational complexity by the number of multiplications for the proposed low-complexity detection in L-OFDM-IM. In the proposed low-complexity detection, calculating $\mathbf{D}$ by Eq. (8) needs $\left(n+\sum_{u=1}^{L} 2 n M(t+k(u-1))\right)$ multiplications. Note that the remaining procedures in $\mathrm{E}$ qs. (10)-(14) only consider the sorting and comparison operations, which generates no multiplication operations. Therefore, the total number of multiplications for the proposed lowcomplexity is $\left(n+\sum_{u=1}^{L} 2 n M(t+k(u-1))\right)$. On the other hand, the total number of the optimal ML detection in Eq. (6) is $\left(2 n\left(2^{\left\lfloor\log _{2} C(t, k)\right\rfloor} M^{k}\right)^{L}\right)$. It should be noted that the conventional OFDM-IM scheme using the optimal ML detection leads to $2 n 2^{\left\lfloor\log _{2} C(n, v)\right\rfloor} M^{k}$ multiplications, which has exactly the same computational complexity as L-OFDMIM using ML detection under the same SEs. To clearly see
TABLE II

MULTIPLICATION COMPARISON OF THE DETECTION METHODS FOR L-OFDM-IM.

\begin{tabular}{|c||c||c||c|}
\hline$(k, t, L, M)$ & Proposed & ML & Reduction (\%) \\
\hline \hline$(1,3,2,2)$ & 68 & 128 & $46.88 \%$ \\
\hline$(2,4,2,4)$ & 294 & 49152 & $99.4 \%$ \\
\hline$(2,4,3,2)$ & 264 & 65536 & $99.6 \%$ \\
\hline
\end{tabular}

the computational complexity reduction of the proposed lowcomplexity detection, we perform the multiplication comparison of two detection methods in Table II. From Table II, we can see that the computational complexity is largely reduced, especially for large values of $k$ and $L$.

\section{Performance AnAlysis And Diversity ENHANCING SCHEME}

\section{A. Upper Bound Analysis}

In this subsection, we analytically derive the upper bound on the BER of L-OFDM-IM systems.

According to (6), the conditional pairwise error probability (CPEP) on $\mathbf{h}_{\alpha}$ is given by

$$
\operatorname{Pr}\left(\mathbf{x}_{\alpha} \rightarrow \hat{\mathbf{x}}_{\alpha} \mid \mathbf{h}_{\alpha}\right)=Q\left(\sqrt{\frac{\left\|\mathbf{H}\left(\mathbf{x}_{\alpha}-\hat{\mathbf{x}}_{\alpha}\right)\right\|^{2}}{2} \gamma}\right)
$$

where $\mathbf{H}=\operatorname{diag}\left\{\mathbf{h}_{\alpha}\right\}$ and $\gamma=1 / N_{0}$ is defined as the signalto-noise ratio (SNR).

With the Q-function approximation [35]

$$
Q(x) \cong \frac{1}{12} e^{-\frac{x^{2}}{2}}+\frac{1}{4} e^{-\frac{2 x^{2}}{3}},
$$

we obtain the unconditional PEP as [19]

$$
\begin{array}{r}
\operatorname{Pr}\left(\mathbf{x}_{\alpha} \rightarrow \hat{\mathbf{x}}_{\alpha}\right)=E_{\mathbf{h}_{\alpha}}\left\{Q\left(\sqrt{\frac{\left\|\mathbf{H}\left(\mathbf{x}_{\alpha}-\hat{\mathbf{x}}_{\alpha}\right)\right\|^{2}}{2} \gamma}\right)\right\} \\
=\frac{1 / 12}{\operatorname{det}\left(\mathbf{I}_{n}+q_{1} \mathbf{K A}\right)}+\frac{1 / 4}{\operatorname{det}\left(\mathbf{I}_{n}+q_{2} \mathbf{K A}\right)},
\end{array}
$$

where $\mathbf{A}=\operatorname{diag}\left\{\mathbf{x}_{\alpha}-\hat{\mathbf{x}}_{\alpha}\right\}^{H} \operatorname{diag}\left\{\mathbf{x}_{\alpha}-\hat{\mathbf{x}}_{\alpha}\right\} ; \mathbf{K}=E\left\{\mathbf{h}_{\alpha} \mathbf{h}_{\alpha}^{H}\right\}$ is the channel covariance matrix; $q_{1}=1 /\left(2 N_{0}\right)$ and $q_{2}=$ $2 /\left(3 N_{0}\right)$.

It can be seen from (17) that $\mathbf{A}$ is a diagonal matrix with $d$ nonzero eigenvalues $\lambda_{1}, \lambda_{2}, \ldots, \lambda_{d}$. Noting that $\mathbf{K}$ is changed into an identity matrix with sufficiently deep interleaving. 
Therefore, the unconditional PEP can be rewritten as

$$
\operatorname{Pr}\left(\mathbf{x}_{\alpha} \rightarrow \hat{\mathbf{x}}_{\alpha}\right) \simeq \frac{1 / 12}{\prod_{t=1}^{d}\left(1+q_{1} \lambda_{t}\right)}+\frac{1 / 4}{\prod_{t=1}^{d}\left(1+q_{1} \lambda_{t}\right)} .
$$

Assuming in a rich scattering environment, (18) can be approximated at high SNR as

$$
\operatorname{Pr}\left(\mathbf{x}_{\alpha} \rightarrow \hat{\mathbf{x}}_{\alpha}\right) \simeq\left(12 q_{1}^{d} \prod_{t=1}^{d} \lambda_{t}\right)^{-1}+\left(4 q_{2}^{d} \prod_{t=1}^{d} \lambda_{t}\right)^{-1}
$$

It is obvious that the diversity order is $d=\min \operatorname{rank}\{\mathbf{A}\}$ [21].

After obtaining the unconditional PEP, an upper bound on the BER is derived according to the union bound as [36]

$$
P_{e} \leq \frac{1}{m 2^{m}} \sum_{\mathbf{x}} \sum_{\hat{\mathbf{x}} \neq \mathbf{x}} N\left(\mathbf{x}_{\alpha} \rightarrow \hat{\mathbf{x}}_{\alpha}\right) \operatorname{Pr}\left(\mathbf{x}_{\alpha} \rightarrow \hat{\mathbf{x}}_{\alpha}\right),
$$

where $N\left(\mathbf{x}_{\alpha} \rightarrow \hat{\mathbf{x}}_{\alpha}\right)$ measures the number of bits in error between $\mathbf{x}_{\alpha}$ and $\hat{\mathbf{x}}_{\alpha}$.

\section{B. Achievable Rate}

We derive the achievable rate of the proposed L-OFDMIM system according to the definition of mutual information between $\mathbf{y}_{\alpha}$ and $\mathbf{x}_{\alpha}$, which is given by [37]

$$
\begin{aligned}
r & =\frac{1}{n} I\left(\mathbf{x}_{\alpha} ; \mathbf{y}_{\alpha}\right)=\frac{1}{n}\left(H\left(\mathbf{x}_{\alpha}\right)-H\left(\mathbf{x}_{\alpha} \mid \mathbf{y}_{\alpha}\right)\right) \\
& =\frac{p}{n}-\frac{1}{n} E_{\mathbf{h}_{\alpha}}\left[H\left(\mathbf{x}_{\alpha} \mid \mathbf{y}_{\alpha}, \mathbf{h}_{\alpha}\right)\right] .
\end{aligned}
$$

The probability density functions (PDFs) of $\mathbf{y}_{\alpha}$ are given by

$$
f\left(\mathbf{y}_{\alpha} \mid \mathbf{x}_{\alpha}, \mathbf{h}_{\alpha}\right)=\frac{1}{\left(\pi N_{0}\right)^{n}} \exp \left(\left\|\mathbf{y}_{\alpha}-\operatorname{diag}\left\{\mathbf{h}_{\alpha}\right\} \mathbf{x}_{\alpha}\right\|^{2}\right),
$$

and

$$
f\left(\mathbf{y}_{\alpha} \mid \mathbf{h}_{\alpha}\right)=\frac{1}{2^{p}} \sum_{\varpi=1}^{2^{p}} f\left(\mathbf{y}_{\alpha} \mid \mathbf{x}_{\alpha}(\varpi), \mathbf{h}_{\alpha}\right),
$$

where $\mathbf{x}_{\alpha}(\varpi)$ denotes the $\varpi$-th observation of $\mathbf{x}_{\alpha}$. By using (22) and (24), there is no closed form for (21). Therefore, we utilize the lower bound technique in [37] to depict the achievable rate as

$$
\begin{aligned}
r \geq \frac{p}{n} & -\left(\log _{2}(e)-1\right) \\
& -\frac{1}{n 2^{p}} \sum_{\varpi=1}^{2^{p}}\left(\log _{2} \sum_{\eta=1}^{2^{p}} \frac{1}{\operatorname{det}\left(\mathbf{I}_{n}+\Xi_{\varpi, \eta}\right)}\right),
\end{aligned}
$$

where $\Xi_{\varpi, \eta}=\frac{1}{2 N_{0}} \operatorname{diag}\left\{\mathbf{x}_{\alpha}(\varpi)-\mathbf{x}_{\alpha}(\eta)\right\}^{H} \operatorname{diag}\left\{\mathbf{x}_{\alpha}(\varpi)-\right.$ $\left.\mathbf{x}_{\alpha}(\eta)\right\}$.

\section{Diversity Enhancing Scheme}

In this subsection, we propose the CI-L-OFDM-IM scheme to provide a diversity gain.

In CI-L-OFDM-IM, we apply the CI design [38] into LOFDM-IM that interleaves the real and imaginary parts of modulated symbols in each layer, such that one modulated symbol is assigned to two different subchannels. First, each signal constellation set is rotated with a certain angle $\theta$ in each layer, which ensures the successful realization of the CI technique. We still take the $\alpha$-th group as an example and the modulated symbols in the $u$-th layer are then updated according to the relation below:

$$
\tilde{\mathbf{s}}_{\alpha}^{u}=e^{j \theta} \mathbf{s}_{\alpha}^{u}=\left[\tilde{s}_{\alpha}^{u, 1}, \tilde{s}_{\alpha}^{u, 2}, \ldots, \tilde{s}_{\alpha}^{u, k}\right]^{T} .
$$

Then, the real and imaginary parts of each modulated symbol are switched with that of its adjacent symbol, which is given by

$$
\mathbf{c}_{\alpha}^{u}=\left[\begin{array}{c}
c_{\alpha}^{u, 1} \\
c_{\alpha}^{u, 2} \\
\vdots \\
c_{\alpha}^{u, k-1} \\
c_{\alpha}^{u, k}
\end{array}\right]=\left[\begin{array}{c}
\mathfrak{R}\left\{\tilde{s}_{\alpha}^{u, 1}\right\}+j \mathfrak{J}\left\{\tilde{s}_{\alpha}^{u, 2}\right\} \\
\mathfrak{R}\left\{\tilde{s}_{\alpha}^{u, 2}\right\}+j \mathfrak{J}\left\{\tilde{s}_{\alpha}^{u, 3}\right\} \\
\vdots \\
\mathfrak{R}\left\{\tilde{s}_{\alpha}^{u, k-1}\right\}+j \mathfrak{J}\left\{\tilde{s}_{\alpha}^{u, k}\right\} \\
\mathfrak{R}\left\{\tilde{s}_{\alpha}^{u, k}\right\}+j \mathfrak{J}\left\{\tilde{s}_{\alpha}^{u, 1}\right\}
\end{array}\right] .
$$

With the SAP $I_{\alpha}^{u}$, the modified signal vector at the $u$-th layer is given by

$$
\chi_{\alpha}^{u}=\left[\begin{array}{c}
\chi_{\alpha}^{u, 1} \\
\chi_{\alpha}^{u, 2} \\
\chi_{\alpha}^{u, 3} \\
\vdots \\
\chi_{\alpha}^{u, n-2} \\
\chi_{\alpha}^{u, n-1} \\
\chi_{\alpha}^{u, n}
\end{array}\right]=\left[\begin{array}{c}
\Re\left\{\tilde{s}_{\alpha}^{u, 1}\right\}+j \mathfrak{J}\left\{\tilde{s}_{\alpha}^{u, 2}\right\} \\
0 \\
\mathfrak{R}\left\{\tilde{s}_{\alpha}^{u, 2}\right\}+j \mathfrak{J}\left\{\tilde{s}_{\alpha}^{u, 3}\right\} \\
\vdots \\
0 \\
\mathfrak{R}\left\{\tilde{s}_{\alpha}^{u, k}\right\}+j \mathfrak{J}\left\{\tilde{s}_{\alpha}^{u, 1}\right\} \\
0
\end{array}\right] .
$$

It can be seen from (26) and (27) that each modulated symbol will traverse through two different subcarriers, which thereby leads to a diversity order of two for CI-L-OFDM-IM systems. Consequently, the resulting signal vector containing all $L$ layers for the $\alpha$-th group is $\chi_{\alpha}=\chi_{\alpha}^{1}+\chi_{\alpha}^{2}+\cdots+\chi_{\alpha}^{L}$. After obtaining $\left\{\chi_{\alpha}\right\}_{\alpha=1}^{g}$ for all groups, the following transmission and detection process is the same as L-OFDM-IM described in Section II.C. Note that the rotation angle $\theta$ will significantly impact on the BER of CI-L-OFDM-IM. One might refer to [20] for more details about the optimal design of rotation angle $\theta$ if interested.

\section{Simulation Results and Discussion}

In this section, we conduct computer simulations to evaluate the BER of L-OFDM-IM systems. For the sake of simplicity, we denote "(CI-)L-OFDM-IM $(k, t, L, M)$, PSK/QAM" as the (CI-)L-OFDM-IM scheme with $n$ subcarriers $(n=t+k(L-$ $1)$ ) for $L$ layers, $k$ active subcarriers out of $t$ subcarriers in each layer and $L$ different $M$-ary PSK/QAM constellations, "(CI-)OFDM-IM $(v, n, M)$, PSK/QAM" as the conventional (CI-)OFDM-IM scheme with $v$ active subcarriers, $n$ total subcarriers and an $M$-ary PSK/QAM constellation. Assume that the $L$ different signal constellations for L-OFDM-IM are set as the rotated PSK/QAM constellations with a rotation angle, and it has been proven that $2 \pi /(L M)$ is the optimal value of the rotation angle [29].

We compare the BER of "L-OFDM-IM $(1,3,2,2)$, PSK", "L-OFDM-IM $(2,6,2,2)$, PSK", "OFDM-IM $(2,4,2)$, PSK" and "OFDM-IM $(4,8,2)$, PSK" in Fig. 3. The simulation curves of "L-OFDM-IM $(1,3,2,2)$, PSK" and "OFDM-IM $(2,4,2)$, PSK" both with an SE of $1 \mathrm{bps} / \mathrm{Hz}$ are drawn in Fig. 3(a). It can be seen from Fig. 3(a) that L-OFDM-IM 


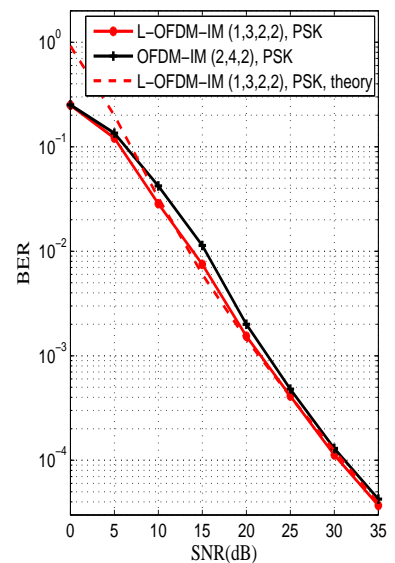

(a) The SE of $1 \mathrm{bps} / \mathrm{Hz}$ for L-OFDM- (b) The SE of $1.25 \mathrm{bps} / \mathrm{Hz}$ for LIM and OFDM-IM. OFDM-IM and OFDM-IM.
Fig. 3. Performance comparison between L-OFDM-IM and OFDM-IM with the same SE.

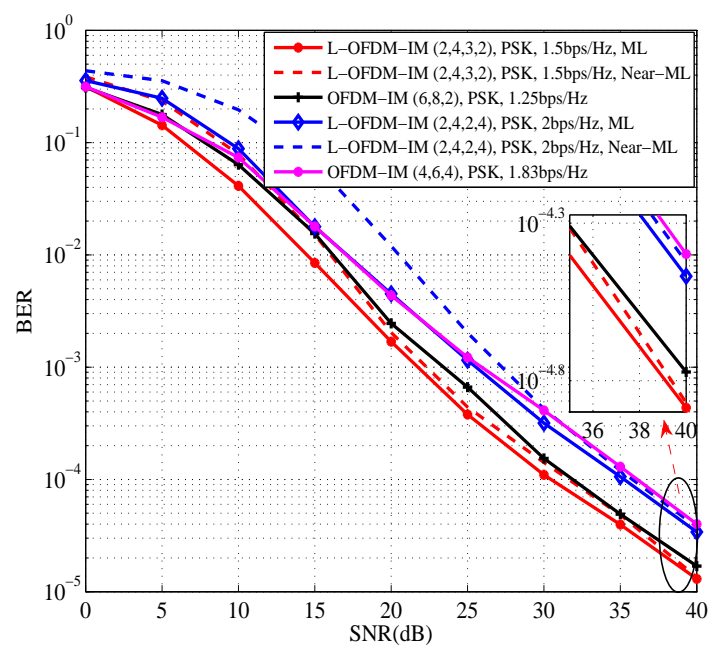

Fig. 4. Performance comparison between L-OFDM-IM and OFDM-IM with different SEs.

performs better than OFDM-IM with a distinct performance gain in the low SNR region while L-OFDM-IM achieves about $0.7 \mathrm{~dB}$ SNR gain than OFDM-IM in the high SNR region. A similar situation also occurs in Fig. 3(b), which demonstrates the BER of "L-OFDM-IM $(2,6,2,2)$, PSK" and "OFDM-IM $(4,8,2)$, PSK" both with $1.25 \mathrm{bps} / \mathrm{Hz}$. Compared to "OFDMIM $(4,8,2)$, PSK", "L-OFDM-IM $(2,6,2,2)$, PSK" obtains circa $2.5 \mathrm{~dB}$ performance gain in the low SNR region and circa $0.8 \mathrm{~dB}$ performance gain in the high SNR region. We hereby summarize that the proposed L-OFDM-IM scheme outperforms the conventional OFDM-IM scheme, especially in the low SNR region. In addition, the theoretical curves of L-OFDM-IM well match the simulation results in the high SNR region in both Figs. 3(a) and 3(b), which verifies the accuracy of the analysis in Section IV.

Fig. 4 presents the BER of "L-OFDM-IM $(2,4,3,2)$, PSK", "L-OFDM-IM $(2,4,2,4)$, PSK", "OFDM-IM $(6,8,2)$, PSK"

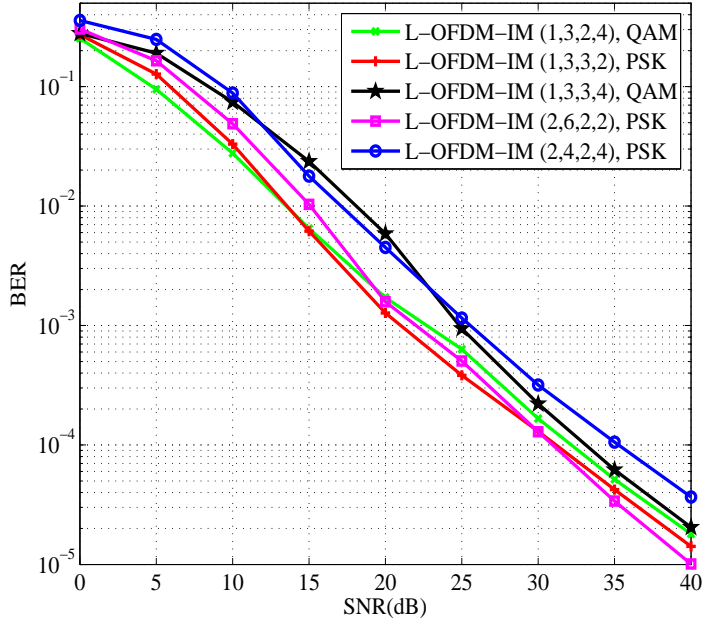

Fig. 5. Diversity comparison among various L-OFDM-IM schemes.

and "OFDM-IM $(4,6,4)$, PSK". Different from Fig. 3, Fig. 4 shows the performance comparison between L-OFDM-IM and OFDM-IM with different SEs. To be specific, "L-OFDM-IM $(2,4,3,2)$, PSK" with $1.5 \mathrm{bps} / \mathrm{Hz}$ achieves an approximate $1.5 \mathrm{~dB}$ SNR gain with respect to "OFDM-IM $(6,8,2)$, PSK" with $1.25 \mathrm{bps} / \mathrm{Hz}$ at $\mathrm{BER}=10^{-4}$ under the same system configuration ( $n=8, v=L k=6)$. Similarly, "L-OFDMIM $(2,4,2,4)$, PSK" with $2 \mathrm{bps} / \mathrm{Hz}$ achieves an approximate $1 \mathrm{~dB}$ SNR gain with respect to "OFDM-IM $(4,6,4)$, PSK" with $1.83 \mathrm{bps} / \mathrm{Hz}$ at $\mathrm{BER}=10^{-4}$ under the same system configuration $(n=6, v=L k=4)$. It should be noted that the proposed L-OFDM-IM scheme with a greater SE obtains a lower BER than that of the conventional OFDM scheme. Additionally, we find that the diversity orders of L-OFDMIM and OFDM-IM are the same, which equals unity. It can be also found that the diversity order of L-OFDM-IM does not change with an increasing number of layers $L$. Fig. 4 also provides the BER comparison between the optimal ML detection and the low-complexity detection for "L-OFDM-IM $(2,4,3,2)$, PSK" and "L-OFDM-IM $(2,4,2,4)$, PSK". It can be seen that the low-complexity detection achieves a worse BER performance than the optimal ML detection in the low SNR region. However, the proposed low-complexity detection approaches the optimal ML detection at the price of negligible SNR loss in the high SNR region. It is concluded that the lowcomplexity detection achieves a near-ML performance with a largely reduced computational complexity.

To further evaluate the diversity order of L-OFDM-IM, we compare the BER of "L-OFDM-IM $(1,3,2,4)$, QAM", "L-OFDM-IM (1, 3, 3, 2), PSK" "L-OFDM-IM (1,3,3,4), QAM", "L-OFDM-IM $(2,6,2,2)$, PSK" and "L-OFDM-IM $(2,4,2,4)$, PSK" in Fig. 5. From Fig. 5, it can be seen that the diversity orders are the same for all cases. By comparing the curves of "L-OFDM-IM $(1,3,2,4)$, QAM" and "L-OFDMIM $(1,3,3,4)$, QAM", it is easy to observe that increasing the number of layers $L$ only will not change the diversity order, which coincides with Fig. 4. Meanwhile, increasing the number of active subcarriers $k$ and the modulation cardinality 


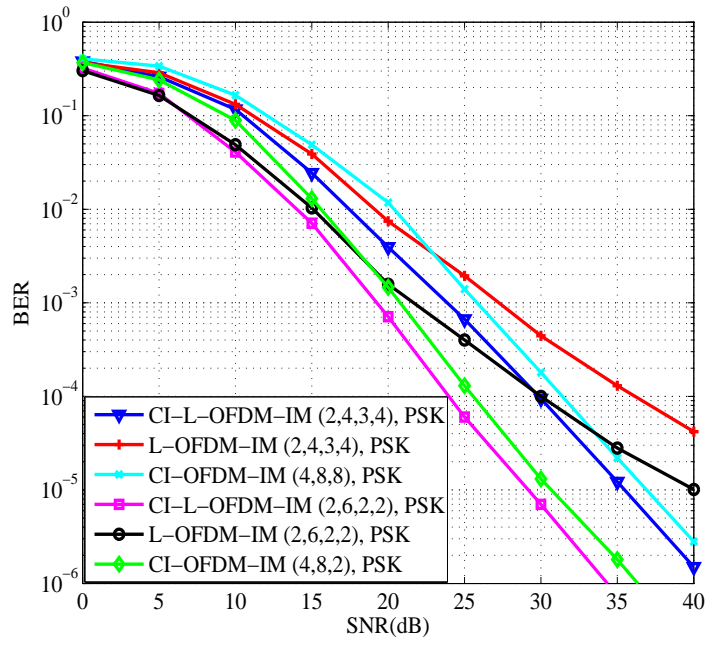

Fig. 6. BER of CI-L-OFDM-IM, L-OFDM-IM and CI-OFDM-IM with different configurations.

$M$ still does not change the diversity order if we compare the curves of "L-OFDM-IM $(2,6,2,2)$, PSK" and "L-OFDM-IM $(2,4,2,4)$, PSK". Moreover, even we change $k$, the number of total subcarriers $t$ and $L$, as can be seen from the curves of " $L$ OFDM-IM $(1,3,3,4)$, QAM" and "L-OFDM-IM $(2,4,2,4)$, PSK", the diversity order also keeps unchanged. Again, similar observations are obtained by comparing the simulation curves of "L-OFDM-IM $(1,3,3,4)$, QAM" and "L-OFDMIM $(2,6,2,2)$, PSK" with different values of parameters $k, t$, $L$ and $M$.

Fig. 6 shows the BER of "CI-L-OFDM-IM $(2,4,3,4)$ ", "CI-L-OFDM-IM $(2,6,2,2)$ ", "L-OFDM-IM $(2,4,3,4)$ ", "LOFDM-IM $(2,6,2,2)$ ", "CI-OFDM-IM $(4,8,8)$ " and "CIOFDM-IM $(4,8,2)$ ". It is noted that we apply PSK for all configurations and $\theta$ is set to $9^{\circ}$ for both CI-L-OFDM-IM and CI-OFDM-IM schemes for simplicity. Due to the CI effect, we see that "CI-L-OFDM-IM $(2,4,3,4)$ " with an SE of $2.25 \mathrm{bps} / \mathrm{Hz}$ obtains a larger performance gain than " $\mathrm{L}$ OFDM-IM $(2,4,3,4)$ " with an SE of $2.25 \mathrm{bps} / \mathrm{Hz}$ in the high SNR region. Compared to the conventional "CI-OFDMIM $(4,8,8)$ " with an SE of $2.25 \mathrm{bps} / \mathrm{Hz}$, "CI-L-OFDM-IM $(2,4,3,4)$ " still obtains a considerable performance gain. Similarly, "CI-L-OFDM-IM $(2,6,2,2)$ " with an SE of $1.25 \mathrm{bps} / \mathrm{Hz}$ is superior to "L-OFDM-IM $(2,6,2,2)$ " and "CI-OFDM-IM $(4,8,2)$ " both with an SE of $1.25 \mathrm{bps} / \mathrm{Hz}$, especially in the high SNR region. It is also obvious that both "CI-L-OFDMIM $(2,4,3,4)$ " and "CI-L-OFDM-IM $(2,6,2,2)$ " achieve a diversity order of two, while "L-OFDM-IM $(2,4,3,4)$ " and "L-OFDM-IM $(2,6,2,2)$ " achieve only unit diversity order, which validates our analysis.

The achievable rates of "L-OFDM-IM $(1,3,2,2)$, PSK", "L-OFDM-IM $(2,6,2,2)$, PSK", "OFDM-IM $(2,4,2)$, PSK" and "OFDM-IM $(4,8,4)$, PSK" are performed in Fig. 7. It can be seen from Fig. 7 that the achievable rates of all schemes increase with the increase of SNR and approach constants in the high SNR region. This is because less detection errors occur at the receiver as SNR increases and the transmitted

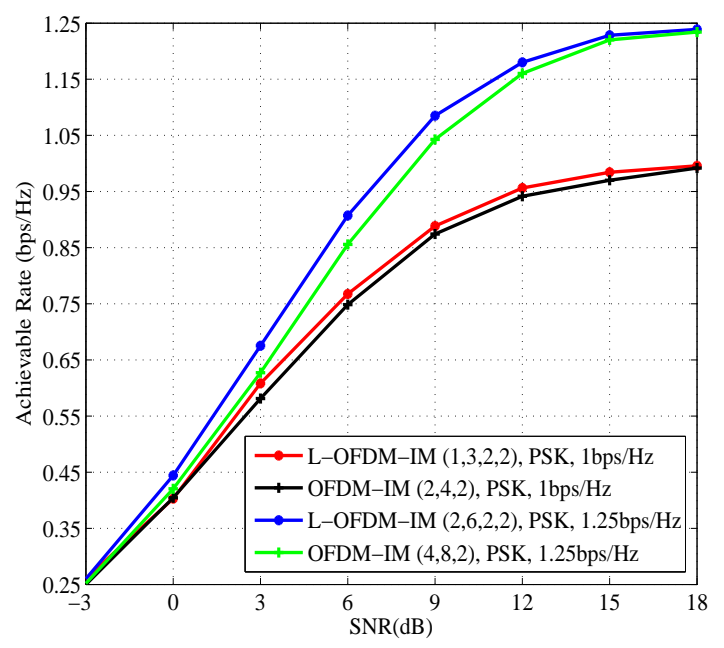

Fig. 7. Achievable rate comparison for L-OFDM-IM and OFDM-IM schemes.

information bits are correspondingly estimated with less errors. More importantly, although the SEs for all schemes tend to saturation at high SNR, we still can see from Fig. 7 that "LOFDM-IM $(1,3,2,2)$, PSK" with $1 \mathrm{bps} / \mathrm{Hz}$ and "L-OFDM-IM $(2,6,2,2)$, PSK" with $1.25 \mathrm{bps} / \mathrm{Hz}$ outperform "OFDM-IM $(2,4,2)$, PSK" with $1 \mathrm{bps} / \mathrm{Hz}$ and "OFDM-IM $(4,8,2)$, PSK" with $1.25 \mathrm{bps} / \mathrm{Hz}$ at low-to-medium SNRs, respectively, which further reveals the advantage of our proposed L-OFDM-IM scheme.

\section{CONCLUSiOnS}

In this paper, we proposed the L-OFDM-IM scheme, which enhances the SE of OFDM-IM without consuming any extra resource. Unlike OFDM-IM, L-OFDM-IM separates all subcarriers into $L$ layers, which select active subcarriers more flexibly. Specifically, the active subcarriers are selected in a layer-wise manner, which increases the length of IM bits and thereby improves the SE. The low-complexity detection method for L-OFDM-IM has been provided to alleviate the detection burden with the negligible performance loss at the high SNR. The BER, achievable rate and diversity order for L-OFDM-IM have been also investigated. In addition, the CIL-OFDM-IM was proposed to improve the diversity order of L-OFDM-IM. Simulation results confirmed that the L-OFDMIM scheme outperforms the conventional OFDM-IM scheme, especially in the low SNR region. Simulations also revealed that the diversity order of L-OFDM-IM dose not change with the number of layers, the number of active subcarriers, the number of candidate subcarriers and the cardinality of constellations. By the numerical results provided, it was also shown that CL-OFDM-IM is capable of obtaining a diversity advantage over L-OFDM-IM.

\section{REFERENCES}

[1] E. Basar, M. Wen, R. Mesleh, M. Di Renzo, Y. Xiao, and H. Haas, "Index modulation techniques for next-generation wireless networks," IEEE Access, vol. 5, pp. 16693-16746, 2017. 
[2] T. Mao, Q. Wang, Z. Wang, and S. Chen, "Novel index modulation techniques: A survey," IEEE Commun. Surveys \& Tutorials, vol. 21, no. 1, pp. 315-348, First Quarter 2019.

[3] X. Cheng, M. Zhang, M. Wen, and L. Yang, "Index Modulation for 5G: Striving to Do More with Less," IEEE Wireless Commun., vol. 25, no. 2, pp. 126-132, Apr. 2018.

[4] M. Wen, X. Cheng, and L. Yang, Index Modulation for 5G Wireless Communications. Berlin, Germany: Springer, 2017.

[5] R. Mesleh, H. Haas, S. Sinanovic, C. W. Ahn, and S. Yun, "Spatial modulation," IEEE Trans. Veh. Technol., vol. 57, no. 4, pp. 2228-2241, July 2008.

[6] J. Jeganathan, A. Ghrayeb, L. Szczecinski, and A. Ceron, "Space shift keying modulation for MIMO channels," IEEE Trans. Wireless Commun., vol. 8, no. 7, pp. 3692-3703, July 2009.

[7] A. Younis, N. Serafimovski, R. Mesleh, and H. Haas, "Generalised spatial modulation," in Proc. 2010 Conf. Record of the 44th Asilomar Conf. on Signals, Syst. and Comput., Pacific Grove, CA, USA, Nov. 2010, pp. 1498-1502

[8] J. Jeganathan, A. Ghrayeb, and L. Szczecinski, "Generalized space shift keying modulation for MIMO channels," in Proc. IEEE 19th Int. Symp. PIMRC, Cannes, France, Sep. 2008, pp. 1-5.

[9] R. Mesleh, S. S. Ikki, and H. M. Aggoune, "Quadrature spatial modulation," IEEE Trans. Veh. Technol., vol. 64, no. 6, pp. 2738-2742, June 2015.

[10] J. Li, M. Wen, X. Cheng, Y. Yan, S. Song, and M. H. Lee, "Generalised pre-coding aided quadrature spatial modulation," IEEE Trans. Veh. Technol., vol. 66, no. 2, pp. 1881-1886, Feb. 2017.

[11] M. Maleki, H. R. Bahrami, and A. Alizadeh, "Layered spatial modulation for multiuser communications," IEEE Trans. Wireless Commun. vol. 15, no. 10, pp. 7143-7159, Oct. 2016.

[12] S. Fang, L. Li, S. Hu, J. Tang, Z. Yue, G. Feng, and A. Pandharipande, "Layered space shift seying modulation over MIMO channels," IEEE Trans. Veh. Technol., vol. 66, no. 1, pp. 159-174, Jan. 2017.

[13] E. Basar, U. Aygolu, E. Panayirci, and H. Poor, "Space-time block coded spatial modulation," IEEE Trans. Commun., vol. 59, no. 3, pp. 823-832, Mar. 2011.

[14] S. Sugiura, S. Chen, and L. Hanzo, "Generalized space-time shift keying designed for flexible diversity-, multiplexing- and complexity-tradeoffs," IEEE Trans. Wireless Commun., vol. 10, no. 4, pp. 1144-1153, Apr. 2011.

[15] Y. Bian, X. Cheng, M. Wen, L. Yang, H. V. Poor, and B. Jiao, "Differential spatial modulation," IEEE Trans. Veh. Technol., vol. 64, no. 7, pp. 3262-3268, July 2015.

[16] L. Xiao, Y. Xiao, P. Yang, J. Liu, S. Li, and W. Xiang, "Space-time block coded differential spatial modulation," IEEE Trans. Veh. Technol., vol. 66 , no. 10 , pp. 8821-8834, Oct 2015.

[17] R. Abu-Alhiga and H. Haas, "Subcarrier-index modulation OFDM,' in Proc. IEEE 20th Int. Symp. Pers., Indoor Mobile R-adio Commun. (PIMRC), Tokyo, Japan, Sep. 2009, pp. 177-181.

[18] D. Tsonev, S. Sinanovic, and H. Haas, "Enhanced subcarrier index modulation (SIM) OFDM," in Proc. IEEE Global Commun. Conf. (GLOBECOM) Workshops, Houston, TX, USA, Dec. 2011, pp. 728-732.

[19] E. Basar, U. Aygolu, E. Panayirci, and H. V. Poor, "Orthogonal frequency division multiplexing with index modulation," IEEE Trans. Signal Process., vol. 61, no. 22, pp. 5536-5549, Nov. 2013.

[20] M. Wen, B. Ye, E. Basar, Q. Li, and F. Ji, "Enhanced orthogonal frequency division multiplexing with index modulation," IEEE Trans. Wireless Commun., vol. 16, no. 7, pp. 4786-4801, July 2017.

[21] M. Wen, Y. Zhang, J. Li, E. Basar, and F. Chen, "Equiprobable subcarrier activation method for OFDM with index modulation," IEEE Commun. Lett., vol. 20, no. 12, pp. 2386-2389, Dec. 2016.

[22] S. Dang, J. P. Coon, and G. Chen, "Adaptive OFDM with index modulation for two-hop relay-assisted networks," IEEE Trans. Wireless Commun., vol. 17, no. 3, pp. 1923-1936, Mar. 2018.

[23] S. Dang, J. Li, M. Wen, S. Mumtaz, and Z. Zhang, "OFDM-IM based dual-hop system using fixed-gain amplify-and-forward relay with preprocessing capability," IEEE Trans. Wireless Commun., vol. 18, no. 4, pp. 2259-2270, Apr. 2019.

[24] Z. Wang, S. Dang, and D. T. Kennedy, "Multi-hop index modulationaided OFDM with decode-and-forward relaying," IEEE Access, vol. 6, pp. 26457-26468, 2018.

[25] B. Zheng, F. Chen, M. Wen, F. Ji, H. Yu, and Y. Liu, "Low complexity ML detector and performance analysis for OFDM with inphase/quadrature index modulation," IEEE Commun. Lett., vol. 19, no. 11, pp. 1893-1896, Nov. 2015.
[26] R. Fan, Y. J. Yu, and Y. L. Guan, "Generalization of orthogonal frequency division multiplexing with index modulation," IEEE Trans. Wireless Commun., vol. 14, no. 10, pp. 5350-5359, Oct. 2015.

[27] E. Basar, "On multiple-input multiple-output OFDM with index modulation for next generation wireless networks," IEEE Trans. Signal Process. vol. 64, no. 15, pp. 3868-3878, Aug. 2016.

[28] T. Mao, Z. Wang, Q. Wang, S. Chen, and L. Hanzo, "Dual-mode index modulation aided OFDM," IEEE Access, vol. 5, pp. 50-60, 2017.

[29] M. Wen, E. Basar, Q. Li, B. Zheng, and M. Zhang, "Multiple-mode orthogonal frequency division multiplexing with index modulation," IEEE Trans. Commun., vol. 65, no. 9, pp. 3892-3906, Sep. 2017.

[30] J. Li, M. Wen, X. Jiang, and W. Duan, "Space-time multiple-mode orthogonal frequency division multiplexing with index modulation," IEEE Access, vol. 5, pp. 23212-23222, 2017.

[31] M. Wen, Q. Li, E. Basar, and W. Zhang, "Generalized multiple-mode OFDM with index modulation," IEEE Trans. Wireless Commun., vol. 17, no. 10, pp. 6531-6543, Oct. 2018.

[32] Q. Li, M. Wen, E. Basar, H. V. Poor, B. Zheng, and F. Chen, "Diversity enhancing multiple-mode OFDM with index modulation," IEEE Trans. Commun., 66, no. 8, pp. 3653-3666, Aug. 2018.

[33] S. Dang, J. Li, M. Wen, and S. Mumtaz, "Distributed processing for multi-relay assisted OFDM with index modulation," IEEE Trans. Wireless Commun., vol. 18, no. 2, pp. 1318-1331, Feb. 2019

[34] Q. Li, M. Wen, Y. Zhang, J. Li, F. Chen, and F. Ji, "Information-guided pilot insertion for OFDM-based vehicular communications systems," IEEE Internet of Things Journal, vol. 6, no. 1, pp. 26-37, Feb. 2019.

[35] M. Chiani and D. Dardari, "Improved exponential bounds and approximation for the Q-function with application to average error probability computation," in Proc. IEEE Global Telecommun. Conf., Bologna, Italy, 2002, pp. 1399-1402.

[36] J. G. Proakis, Digital Communications, 3rd ed. New York: McGraw-Hill, 1995.

[37] M. Wen, X. Cheng, M. Ma, B. Jiao, and H. V. Poor, "On the achievable rate of OFDM with index modulation," IEEE Trans. Signal Process., vol. 64, no. 8, pp. 1919-1932, Apr. 2016.

[38] E. Basar, "OFDM with index modulation using coordinate interleaving," IEEE Wireless Commun. Lett., vol. 4, no. 4, pp. 381-384, Aug. 2015.

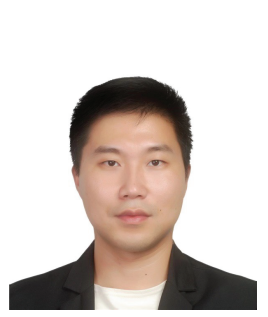

Jun Li (S'13-M'17) received the M.S. and the Ph.D. degrees from Chonbuk National University, Jeonju, South Korea, in 2011 and 2016, respectively. He has been an Assistant Professor with Guangzhou University, Guangzhou, China. He had participated in the World Class University (WCU) project, sponsored by the National Research Foundation (NRF) of Korea grant funded by the Korean Ministry of Education Science and Technology (MEST) as Vice Head Researcher. His research interests include spatial modulation and OFDM index modulation.

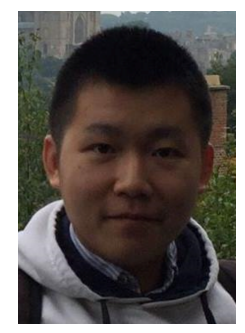

Shuping Dang (S'13-M'18) received B.Eng (Hons) in Electrical and Electronic Engineering from the University of Manchester (with first class honors) and B.Eng in Electrical Engineering and Automation from Beijing Jiaotong University in 2014 via a joint ' $2+2$ ' dual-degree program. He also received D.Phil in Engineering Science from University of Oxford in 2018. Dr. Dang joined in the R\&D Center, Huanan Communication Co., Ltd. after graduating from University of Oxford and is currently working as a Postdoctoral Fellow with the Computer, Electrical and Mathematical Science and Engineering Division, King Abdullah University of Science and Technology (KAUST). He serves as a reviewer for a number of key journals in communications and information science, including IEEE TRANSACTIONS ON WIRELESS COMMUNICATIONS, IEEE TRANSACTIONS ON COMMUNICATIONS and IEEE TRANSACTIONS ON VEHICULAR TECHNOLOGY. His current research interests include artificial intelligence assisted communications, novel modulation schemes and cooperative communications. 


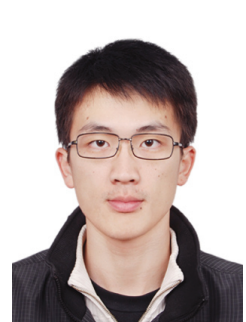

Miaowen Wen (M'14-SM'18) received his Ph.D. degree in Signal and Information Processing from Peking University, China, in 2014. From 2012 to 2013, he was a Visiting Student Research Collaborator with Princeton University, USA. He is currently an Associate Professor with the South China University of Technology, China, and a Post-doctoral Fellow with The University of Hong Kong, China. He has published over 100 research papers, including over 60 journal papers. His research interests include index modulation, non-orthogonal multiple access, physical layer security, and molecular communications.

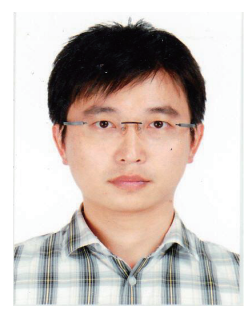

Xueqin Jiang (M'12) received the B.S degree from Nanjing Institute of Technology, Nanjing, Jiangsu, P.R. China, in computer science. He received the M.S and Ph.D degree from Chonbuk National university, Jeonju, Korea, in electronics engineering. He is now an Associate Professor at School of Information Science and Technology, Donghua University, Shanghai, China. He is the author/coauthor of more than 70 technical papers, several book chapters. His main research interests include LDPC codes, physical-layer security and wireless

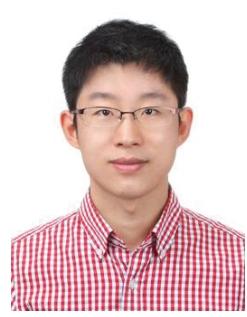

Yuyang Peng (M'16) received M.S. and Ph.D. degrees in electrical and electronic engineering from Chonbuk National University, Jeonju, South Korea, in 2011 and 2014, respectively. From 2014 to 2018 , he has been a postdoctoral research fellow with Korea Advanced Institute of Science and Technology (KAIST), Daejeon, South Korea. He is currently an Assistant Professor with the Faculty of Information Technology at Macau University of Science and Technology (MUST), Taipa, Macau. His research activities lie in the broad area of digital communications, wireless sensor networks, and computing. In particular, his current research interests include cooperative communications, energy optimization, and cloud computing.

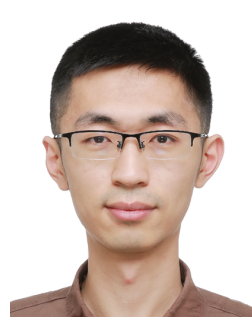

Han Hai received his B.S. degree in information and computing sciences from South Central University for Nationalities, P.R. China, and the M.S. and the $\mathrm{Ph} . \mathrm{D}$. degrees in electronics engineering from Chonbuk National University, Korea, in 2013 and 2018, respectively. He is currently an assistant professor at Donghua University, Shanghai, China. His research interests include wireless communications, MIMO and spatial modulation

communications. 